\begin{tabular}{|c|c|c|}
\hline 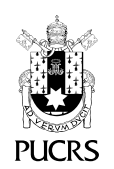 & $\begin{array}{l}\text { ESCOLA DE CIÊNCIAS } \\
\text { DA SAÚDE E DA VIDA }\end{array}$ & $\begin{array}{l}\text { Psico, Porto Alegre, v. 52, n. 3, p. 1-15, jul.-set. } 2021 \\
\text { e-ISSN: 1980-8623 | ISSN-L: 0103-5371 }\end{array}$ \\
\hline d) http: & doi.org/10.15448/1980-8623.2021.3.41302 & \\
\hline
\end{tabular}

\title{
Impactos do avanço da pandemia de COVID-19 na saúde mental de profissionais de saúde
}

\author{
Impacts of COVID-19 pandemic advancement on healthcare workers mental health \\ Impactos del avance de la pandemia COVID-19 en la salud mental de los profesionales \\ sanitarios
}

Pricila Cristina Correa Ribeiro ${ }^{1}$ orcid.org/0000-0001-9431-2707 priccr@gmail.com

Marco Antônio Silva Alvarenga ${ }^{2}$ orcid.org/0000-0002-1168-5733 alvarenga@ufsj.edu.br

Tiago Geraldo de Azevedo ${ }^{2}$ orcid.org/0000-0002-0392-3463 tiagoaz777@gmail.com

Paulo Felipe Ribeiro Bandeira orcid.org/0000-0001-8260-0189 paulo.bandeira@urca.br

Elder Gomes Pereira ${ }^{1}$ orcid.org/0000-0002-6368-5698 elder.gomespsi@gmail.com

Marcela Mansur-Alves ${ }^{1}$ orcid.org/0000-0002-3961-3475. marmansura@gmail.com

Juliana Alvares-Teodoro' orcid.org/0000-0002-0210-0721 jualvares@gmail.com

Camila Batista Peixoto ${ }^{1}$ orcid.org/0000-0002-6999-9460 camimilabp@gmail.com

Marina Luiza Nunes Diniz ${ }^{1}$ orcid.org/0000-0002-1198-1858 marinaluizanunesd@gmail.com

Sabrina Kelly Pessoa de Freitas ${ }^{1}$ orcid.org/0000-0001-7001-9354 sabrinafreitas.psico@outlook.com

Maycoln Leôni Martins Teodoro ${ }^{1}$ orcid.org/0000-0002-3021-8567 mlmteodoro@hotmail.com

Recebido em: 12 jul. 2021. Aprovado em: 28 jul. 2021 Publicado em: 27 out. 2021

\section{(c) (i)}

Artigo está licenciado sob forma de uma licença Creative Commons Atribuição 4.0 Internacional.
Resumo: O estudo verificou a associação e a influência entre variáveis sociodemográficas, laborais, impactos da pandemia (desesperança, contaminação, óbito na família), traços de personalidade e de saúde mental em profissionais de saúde brasileiros em dois tempos distintos da pandemia de COVID-19. Foram incluídos 155 profissionais que responderam um questionário online. Foi utilizado o modelo de redes para a análise dos dados. Os sintomas de depressão, ansiedade e estresse foram os mais influentes no modelo testado e apresentaram associações com a desesperança nos dois tempos. No tempo 1, o estigma foi uma das variáveis mais influentes. No tempo 2, o estigma e a ansiedade reduziram sua influência, enquanto o estresse e a desesperança tornaram-se mais influentes. Os alvos das intervenções para os profissionais de saúde podem ser diferenciados no início e no avanço do contexto pandêmico, mas cabe contínua focalização do estresse e da desesperança. Palavras-chave: pandemia COVID-19, saúde mental, trabalhadores da saúde, análise da complexidade

Abstract: The study verified the association and influence between sociodemographic and labor variables, pandemic impacts (hopelessness, contamination, death in the family), personality and mental health traits in Brazilian health professionals at two different times of the COVID-19 pandemic. There were 155 professionals included who replied to an online questionnaire. The network model was used for data analysis. Symptoms of depression, anxiety, and stress were the most influential variables in the model tested and showed connections with hopelessness at both times. At time 1, stigma was one of the most influential variables. At time 2 , stigma and anxiety reduced their influence, while stress and hopelessness became more prominent. The targets of interventions for health professionals can be differentiated in relation to the onset and progression of the pandemic context, but with a continuous focus on the level of stress and hopelessness for intervention. Keywords: COVID-19 pandemics, mental health, healthcare workers, systems analysis

Resumen: El estudio verificó la asociación e influencia entre variables sociodemográficas y laborales, impactos de la pandemia (desesperanza, contaminación, muerte en la familia), rasgos de personalidad y salud mental en trabajadores sanitarios brasileños en dos momentos de la pandemia del COVID-19. Se incluyeron 155 profesionales que respondieron a un cuestionario en línea. Se utilizó el modelo de red para el análisis. Los sintomas de depresión, ansiedad y estrés fueron las variables más influyentes y mostraron conexiones con la desesperanza en ambos momentos. En el momento 1, el estigma fue una de las variables más influyentes. En el momento 2, el estigma y la ansiedad redujeron su influencia y el estrés y la desesperanza se hicieron más prominentes. Los objetivos de las intervenciones para los trabajadores sanitarios se pueden diferenciar en el inicio y en el avance de la pandemia, pero es necesario un enfoque continuo en el estrés y desesperanza. Palabras clave: pandemia de COVID-19, salud mental, trabajadores sanitarios, análisis de sistemas

\footnotetext{
Universidade Federal de Minas Gerais (UFMG), Belo Horizonte, MG, Brasil.

Universidade Federal de São João del-Rei (UFSJ), São João del-Rei, MG, Brasil.

Universidade Regional do Cariri (URCA), Crato, CE, Brasil.
} 
Desde o início da pandemia de COVID-19, decretada em março de 2020 (WHO, 2020), os riscos para o sofrimento mental entre os profissionais de saúde foram reconhecidos em decorrência das adversidades do trabalho neste contexto (Lancet, 2020). Evidenciou-se a presença de altos indices de ansiedade, estresse e depressão nesta população (Lai et al., 2020), além dos sintomas psicossomáticos, do medo de se infectar e da infecção dos familiares (Teixeira et al., 2020).

Os profissionais que atuam na linha de frente, aqueles envolvidos no diagnóstico e no tratamento aos pacientes com suspeita ou confirmação de COVID-19, foram descritos como mais afetados pelo sofrimento mental que outros grupos profissionais (Lai et al., 2020; Lu et al., 2020). Com a avanço da pandemia, fatores como a exaustão física e emocional (Avanian, 2020) e a desesperança (Hacimusalar et al., 2020) tornam-se ainda mais relevantes quanto ao seu efeito sobre a saúde mental. Além disso, fatores comuns à população geral, como a perda de renda familiar e a sobrecarga com a oferta de suporte aos mais vulneráveis, também foram associados ao prejuizo da saúde mental dos profissionais de saúde (Lancet, 2020).

No Brasil, o primeiro caso de COVID-19 foi confirmado em 26 de fevereiro e o primeiro óbito em 17 de março de 2020 (Ministério da Saúde, 2020). Neste periodo, o controle da pandemia foi centralizado na paralisação de atividades não essenciais, recomendações de distanciamento social, higienização das mãos e o uso de máscaras, o que foi considerado efetivo para a redução do número de infectados (Canabarro et al., 2020). Após um periodo de declínio ou estabilidade, o número de novos casos e óbitos voltou a crescer a partir de outubro de 2020. Contudo, diferente do início da pandemia, adotou-se menor rigor das medidas de controle o que contribuiu para o colapso do sistema de saúde em alguns estados, como no Amazonas (Moraes, 2021). O avanço da pandemia de COVID-19 no Brasil foi agravado pela falta de consenso entre os governantes quanto às diretrizes de controle da doença, pelas disputas políticas e respostas insuficientes para os prejuízos socioeconômicos no país (Pêgo et al., 2020; Bueno et al., 2021).
Pelo exposto, a pandemia de COVID-19 é reconhecida como um cenário adverso no qual fatores contextuais, como condições laborais e de controle epidemiológico, e individuais, como condições psicológicas, interagem formando um sistema complexo, que pode ser visto como auto-organizado, dinâmico e não linear (Langellier et al., 2018), capaz de influenciar a saúde mental de profissionais de saúde. Assim, o estudo teve como objetivo associar variáveis sociodemográficas, do trabalho habitual e de saúde mental em profissionais de saúde durante dois momentos distintos da pandemia de COVID-19 a partir de uma perspectiva de redes.

\section{Método}

\section{Desenho de estudo}

A partir de um estudo transversal sobre a saúde mental de adultos, que reuniu dados de 560 profissionais de saúde no Brasil, durante o periodo inicial da pandemia no Brasil (Tempo 1), foi delineada uma pesquisa longitudinal (Tempo 2) que reavaliou 151 profissionais de saúde. A primeira aplicação dos instrumentos foi realizada em abril de 2020 (Tempo 1) e a segunda aplicação (Tempo 2) ocorreu a partir da segunda quinzena de outubro até a primeira semana de dezembro de 2020. O Tempo 1 coincide com o primeiro mês de adoção de isolamento social como estratégia de controle da pandemia, e o Tempo 2 com um novo aumento no número de infectados, após um periodo de estabilidade ou redução dos casos, na maior parte do País.

\section{Participantes}

Para a captação da amostra no Tempo 1 utilizou-se a estratégia bola de neve, com disparo, via redes sociais digitais, da divulgação do estudo e link para acesso ao questionário a ser respondido remotamente. No Tempo 2, foi enviado e-mail para os participantes com o convite para a segunda participação e o link de acesso ao questionário. Um total de 151 participantes responderam aos questionários do tempo 1 e 2 e constituíram a amostra do presente estudo caracterizada pela média de idade de 39,87 (DP=10,51) anos; 90,7\% 
de mulheres; $49,3 \%$ de profissionais psicólogos, $46,81 \%$ médicos, $24,16 \%$ enfermeiros e o restante distribuidos em outras áreas profissionais da saúde. A pesquisa foi aprovada por Comitê de ética e pesquisa da Universidade Federal de Minas Gerais sob o registro 07077019.3.0000.5149.

\section{Instrumentos}

Os instrumentos e as variáveis que foram incluidas nos questionários respondidos pelos participantes, no tempo 1 e 2, estão descritos na Tabela 1.

Tabela 1 - Descrição dos Instrumentos, Variáveis e Tempos (1 ou 2) em que foram aplicados estes instrumentos no estudo

\section{Instrumentos}

\section{Descrição das variáveis (Tempo de aplicação)}

Questionário sociodemográfico e de condições de trabalho durante a pandemia

Traços de personalidade, avaliados com a versão brasileira do Big Five Inventory, ([IGFP-5] John et al., 1991; Andrade, 2008)

Questionário de Regulação da Emoção ([QRE] Gross \& John, 2003; Gouveia et al., 2018) que mede reavaliação cognitiva $(\alpha=0,67)$, redirecção da atenção $(\alpha=0,63)$ e supressão emocional $(\alpha=0,64)$

Depression, Anxiety, and Stress Scale ([DASS-21] Vignola \& Tucci, 2014; Lovibond \& Lovibond, 1996). Os alphas de Cronbach para a versão brasileira da DASS-21 foram de 0.92 (subescala depressão); 0,90 (subescala estresse) e 0,86 (subescala ansiedade)

Inventário de Percepção do Estigma em Relação ao Profissional de Saúde ([PERPS] elaborado pelos próprios autores)

Frequency of Suicidal Ideation Inventory ([FSII] Chang \& Chang, 2016). A versão brasileira, FSII-Br, apresenta um único fator com alpha de Cronbach igual a 0,88 (Teodoro et al., 2020)

Escala de Estresse Percebido ([PSS] Cohen et al., 1983) A versão brasileira do PSS apresenta um nivel satisfatório de consistência interna $(\alpha=0,83$, Dias et al., 2015).
Idade; sexo; profissão; situação conjugal (Tempo 1).

Perda de renda após início da pandemia; manutenção de trabalho presencial; carga horária semanal de trabalho; se atuava na linha de frente no enfrentamento a Covid-19; disponibilidade e necessidade de Equipamentos de Proteção Individual. (Tempo 1 e 2).

Fatores neuroticismo, extroversão e conscienciosidade, compostos por 24 itens do IGFP-5, para os quais foram obtidos os coeficientes de consistência interna 0,89; 0,70 e 0,64, respectivamente. Os itens são respondidos com uma escala que varia de 1 (nunca) a 5 (frequentemente) (Tempo 1).

Escore total composto pelos 10 itens do QRE, respondidos em escala Likert de 7 pontos, que varia de 1 (discordo totalmente) a 7 (concordo totalmente) A consistência interna foi 0,856 (Tempo 1).

Sintomas de depressão, sintomas de ansiedade e sintomas de estresse obtidos com as subescalas da DASS-21, que é composta de 21 itens, medidos em escala Likert de 4 pontos. Os coeficientes obtidos foram iguais a 0,89; 0,87 e 0,87 para as subescalas de estresse, ansiedade e depressão, respectivamente, no tempo 1; e 0,81; 0,77 e 0,70 para estresse, depressão e ansiedade, respectivamente, no tempo 2. (Tempo 1 e 2).

Escore total da PERPS obtido com as respostas para 5 afirmativas (exemplo item 1: "Eu penso que a maioria das pessoas têm evitado tocar em mim porque eu sou profissional de saúde"), obtidas em escala do tipo Likert de 5 pontos, que varia de 1 (discordo fortemente) a 5 (concordo fortemente). Os indices de consistência interna foram 0,87 para o tempo 1 e 0,83 para o tempo 2 (Tempo 1 e 2).

Escore total de ideação obtido com o FSII-Br, composto por 5 itens que avaliam a frequência de ideação suicida nos últimos 12 meses por meio de uma escala Likert de 5 pontos que varia de 1 ("nunca") a 5 ("quase todos os dias"). Para esta pesquisa os índices de consistência interna foram iguais a 0,89 e 0,92 para o tempo 1 e 2, respectivamente. (Tempo 1 e 2).

Percepção de manejo positivo do estresse foi obtida com os itens 4, 5 , 6, 7, 9, 10 e 13 e percepção de manejo negativo com os itens $1,2,3,8$, 11, 12 e 14 da PSS, respondidos em escala Likert de 5 pontos, que varia entre o (nunca) e 4 (muito frequente). Os alfas de Cronbach foram 0,79 e 0,87 para manejo positivo do estresse, e 0,85 e 0,88 para manejo negativo para o tempo 1 e 2 , respectivamente (Tempo 1 e 2). 
Escala de Satisfação com a Vida (ISWLS-BP] Diener et al., 1985). A versão brasileira da SWLS-BP apresenta indice de confiabilidade adequado (Alpha = 0,81) (Gouveia et al., 2009

Questionário com perguntas estruturadas sobre desfechos da pandemia Covid-19
Escore de satisfação com a vida obtido com os 5 itens da SWLS-BP, em escala Likert de 7 pontos, que varia de 1 (discordo totalmente) a 7 (concordo totalmente). A consistência interna foi 0,92 (Tempo 1 e 2).

Desesperança percebida em relação à pandemia (tempo 1 e 2); diagnostico de Covid-19; algum membro da familia ser diagnosticado com Covid-19; alguém da familia faleceu devido a Covid-19 (Tempo 2).

\section{Análise de dados}

Os dados obtidos foram analisados por meio do software IBM Statistical Package for Social Sciences (SPSS), versão 26, e o programa JASP para IOS, versão 0.14, com a finalidade de traçar graficamente as redes e calcular seus ajustes. A análise de frequência foi aplicada às variáveis sociodemográficas e relacionada ao contexto de trabalho e as descritivas para idade, horas de trabalho e escores dos instrumentos utilizados associadas à diferença de média com tamanho dos efeitos (Field, 2013). Foi conduzida análise de rede para avaliar as associações entre variáveis sociodemográficas, laborais, individuais e da saúde mental e verificar quais são mais influentes dentro de um sistema complexo.

A perspectiva de redes é uma técnica de análise de dados para avaliar sistemas complexos, portanto, pode-se compreender o padrão emergente de um sistema com variáveis de diferentes naturezas que interagem de forma não linear e, consequentemente, melhorar o processo de intervenção a partir das variáveis mais influentes. $O$ indicador de centralidade foi relatado para identificar as variáveis mais influentes na rede. O algoritmo Fruchterman-Reingold foi aplicado para que os dados fossem apresentados no espaço relativo no qual as variáveis com associação mais forte permanecem juntas e com relações menos fortes se repelem (Fruchterman \& Reingold, 1991). Para melhorar a precisão da rede, utilizou-se o modelo de campos aleatórios de Markov em pares. $\mathrm{O}$ algoritmo adiciona uma penalidade L1 (regressão de vizinhança regularizada). A regulação é estimada por um operador de seleção e contração menos completo (LASSO) que controla a rede esparsa.

Foi observado o critério de informação Bayesiana estendida (EBIC) para selecionar o Lambda do parâmetro de regularização. O EBIC usa um hiperparâmetro (y) que determina a esparsidade dos modelos (Foygel \& Drton, 2011). O valor de y foi determinado em 0,50 (variação de o a 0,50). A análise de rede usa algoritmos regularizados de redução absoluta e operador de seleção (LASSO) para obter a matriz de precisão (matriz de peso). Quando padronizada, essa matriz representa as correlações entre as variáveis da rede. A rede é apresentada em um gráfico que inclui as variáveis (nós) e as relações (linhas). As linhas de cor azul representam associações positivas e as linhas de cor vermelha representam associações negativas. A espessura e a intensidade das linhas representam a magnitude das associações. Os pequenos efeitos (sensibilidade às condições iniciais) em sistemas complexos são considerados fundamentais para este tipo de análise, portanto a decisão sobre a magnitude de associação entre as variáveis presentes no modelo parte de uma escolha teórica (Caldarelli, 2020).

\section{Resultados}

Os dados descritivos informam que as medidas aplicadas nos dois tempos não apresentaram diferença de média significativa, com exceção da percepção de estigma em relação ao profissional de saúde, que diminuiu no tempo 2 comparada ao tempo 1, com tamanho de efeito moderado $(r=0,23)$ (ver Tabela 2). 
Tabela 2 - Análise de frequência, diferença de média e cálculo do tamanho do efeito para as variáveis coletadas no Tempo 1 e Tempo 2 do Estudo

\begin{tabular}{|c|c|c|c|c|c|c|c|c|}
\hline \multirow[t]{2}{*}{ Variáveis } & \multicolumn{3}{|c|}{ Tempo 1} & \multicolumn{3}{|c|}{ Tempo 2} & \multicolumn{2}{|c|}{$\begin{array}{c}\text { Diferença } \\
\text { de média\# e } \\
\text { tamanho de efeito }\end{array}$} \\
\hline & $\%$ & M & DP & $\%$ & M & DP & $\mathbf{p}$ & $\mathbf{r}$ \\
\hline Atuação na Linha de Frente & 50,33 & & & 38,41 & & & & \\
\hline Afetado pela pandemia & 56,29 & & & 58,94 & & & & \\
\hline Receio da Sobrecarga de trabalho & 33.77 & & & 42,38 & & & & \\
\hline EPI insuficiente para o trabalho & 30,60 & & & 21,20 & & & & \\
\hline EPI suficiente & 25,80 & & & 45,00 & & & & \\
\hline $\begin{array}{l}\text { Medo de Contaminação pelo Coronavírus } \\
\text { Medo de se contaminar }\end{array}$ & 88,70 & & & 82,11 & & & & \\
\hline Medo de contaminar outras pessoas & 81,45 & & & 90,06 & & & & \\
\hline Medo de algum familiar se contaminar & 81,50 & & & 86,75 & & & & \\
\hline Diagnosticado com Covid-19 & - & & & 14,56 & & & & \\
\hline Perdeu algum familiar devido a Covid-19 & & & & 6,62 & & & & \\
\hline Carga horária de trabalho semanal & & 32,39 & 15.52 & & 28,87 & 21,07 & - & - \\
\hline PERPS & & 10,14 & 5.94 & & 7,78 & 3.52 & 0,000 & 0,23 \\
\hline SWSL-BP & & 23.55 & 7,49 & & - & - & - & - \\
\hline QRE & & 41,72 & 12,61 & & - & - & - & - \\
\hline Neuroticismo - IGFP-5 \#\# & & 22,78 & 4,47 & & - & - & - & - \\
\hline Extroversão - IGFP-5 \#\# & & 27,95 & 7,02 & & - & - & - & - \\
\hline Conscienciosidade - IGFP-5 & & 29,26 & 4,99 & & - & - & - & - \\
\hline Subescala Depressão - DASS-21 & & 5,85 & 5,02 & & 5.58 & 4,46 & 0,710 & - \\
\hline Subescala Ansiedade - DASS-21 & & 4,99 & 5,16 & & 4,11 & 3,49 & 0,141 & - \\
\hline Subescala Estresse - DASS-21 & & 9,23 & 5,30 & & 9,07 & 4.77 & 0,803 & - \\
\hline PSS - Enfrentamento positivo do estresse & & 22,94 & 4,83 & & 22,48 & 5,64 & 0,288 & - \\
\hline PSS - Enfrentamento negativo do estresse & & 22,58 & 6,12 & & 22,08 & 6.59 & 0,300 & - \\
\hline FISS & & 5,81 & 2,57 & & 5,83\#\# & 2,56 & 0,701 & - \\
\hline
\end{tabular}

Nota: $M$ = média; DP = igual a desvio padrão; \# teste de diferença de média não paramétrico de Wilcoxon (mesma amostra); $p$ = nivel significância; $r=$ tamanho de efeito baseado no $r$ de Pearson (baixo < 0,2; moderando $\geq 0,2$ e < 0,5; grande $\geq 0,5 ; \# \# N$ = 142; Afetado pela pandemia = Sentindo-se afetado pela pandemia, apesar de bem a maior parte do tempo; EPI = Equipamento de Proteção Individual; PERPS = Percepção do Estigma em relação ao profissional de saúde; SWSL-BP = Satisfação com a Vida; QRE = Regulação Emocional; IGFP-5: Escala de Personalidade DASS-21= Escala de depressão, ansiedade e estresse; PSS= Percepção de enfrentamento do estresse; FISS= ideação suicida.

Inicialmente foram comparadas as variáveis mensuradas idênticas aos dois tempos. A rede apresentou alterações em sua topologia, ou disposição, entre os tempos 1 e 2 da coleta de dados. A Figura 1a apresenta a rede EBIC $(y=0.50)$ com as associações entre as variáveis medidas no tempo 1. Nos principais resultados do tempo 1 verificou-se associações positivas entre ansiedade e es- tresse (0,33); horas de trabalho e atuação na linha de frente $(0,32)$; sintomas depressivos e ansiedade $(0,29)$ e estresse $(0,24)$; desesperança percebida e estigma e sintomas depressivos, ambas iguais a 0,27; percepção de enfrentamento negativo do estresse e maiores niveis de estresse $(0,27)$ e ideação suicida e sintomas de depressão $(0,21)$. 
a

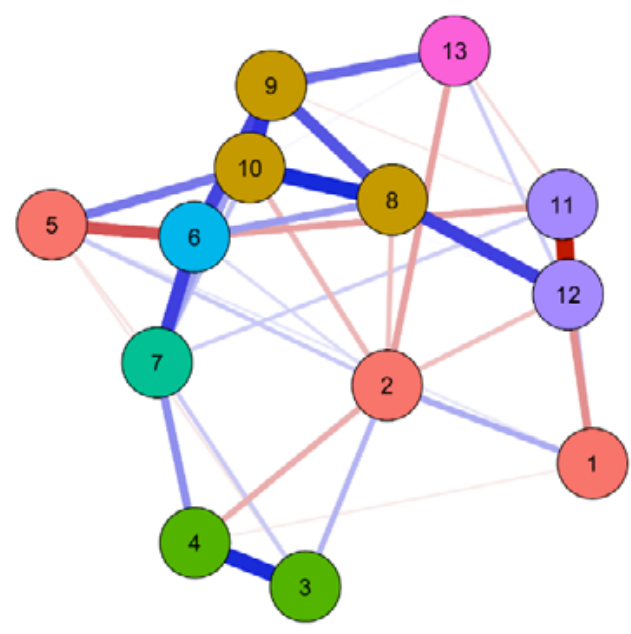

Sociodemográficas

1: idade

- 5: Quedrenda

DASS21

DASS21

- 9: DASSdt1

- 10: DASSat1

Contexto de Trabalho

- 3: horatrab

- 4: linhafrente

Estigma

- 7: ESTetott1

Desesperança

- 6: preocdesesp

Estresse

- 11: eeptpost1

12: eeptnegt

Ideaçấo Suicida

- 13: eistott1

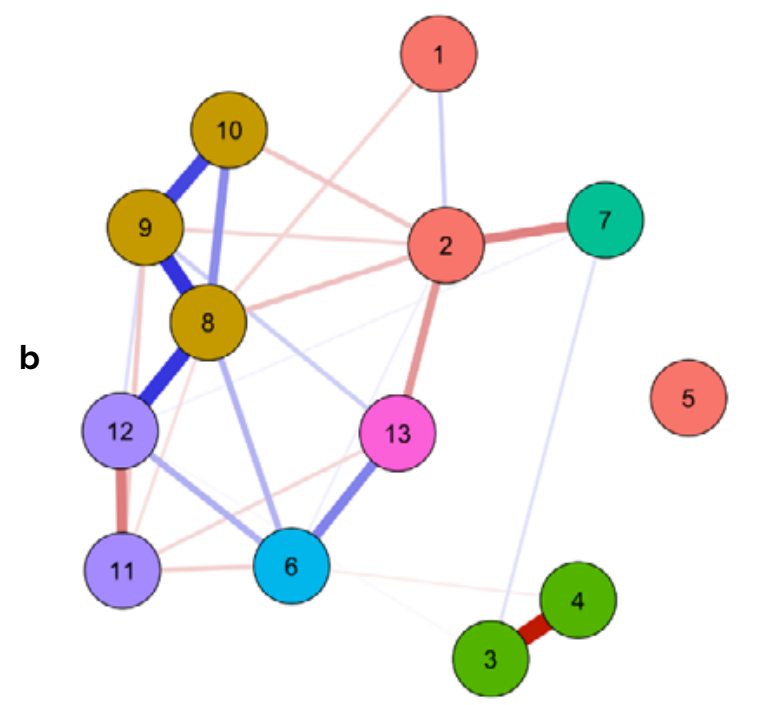

Sociodemográfica

- 1: idade

2. sexo

DASS21

DASS21

8: DASSestt2

9: DASSdt12

Contexto de Trabalhe

-3. hotrabsemt2

- 4: linfrentt2

Estigma

- 7: ESTetott2

Desesperança

6: preo8dupant2

Estresse

12: eeptnegt2

Ideaçāo Suicida

- 13: eistott2

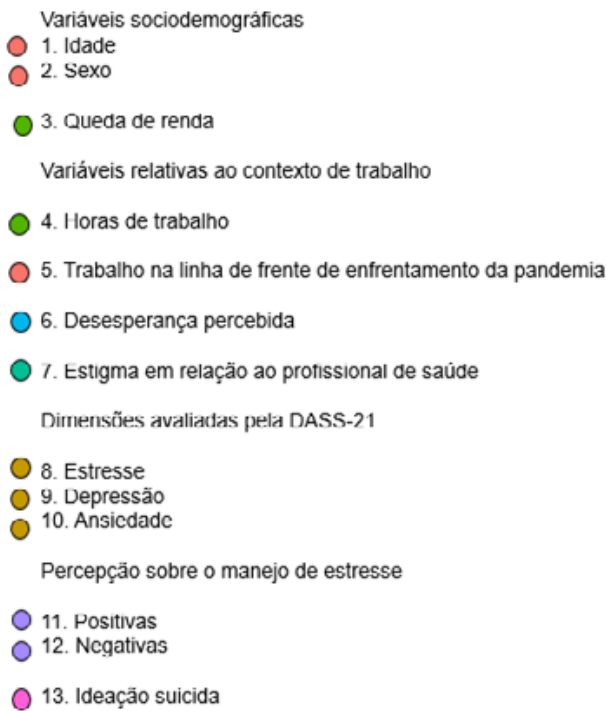

Figura 1 - Rede EBIC y (0.50) das associações para o Tempo 1(a) e 2(b) entre fatores sociodemográficos, contextuais laborais, desesperança percebida, estigma em relação ao profissional de saúde, ansiedade. estresse e depressão, estratégia de enfrentamento do estresse, ideação suicida em profissionais de saúde. 
As associações negativas mais fortes foram entre a perda de esperança e a queda de renda $(-0,24)$, e a diminuição da percepção positiva de enfrentamento do estresse e o aumento da percepção negativa $(-0,36)$ (ver Tabela 3). As variáveis de maior influência neste modelo foram os niveis de depressão, ansiedade e estresse, 1,573, 1,254 e 1,138 respectivamente, o estigma percebido em relação ao profissional de saúde (0,867), a desesperança percebida $(0,302)$ e o número de horas trabalhadas $(0,275)$ (ver tabela 6$)$.

Tabela 3 - Matriz de pesos das variáveis semelhantes ao tempo 1 e 2 do estudo

\section{Tempo 1}

\begin{tabular}{|c|c|c|c|c|c|c|c|c|c|c|c|c|c|}
\hline & 1 & 2 & 3 & 4 & 5 & 6 & 7 & 8 & 9 & 10 & 11 & 12 & 13 \\
\hline 1 & 0 & & & & & & & & & & & & \\
\hline 2 & 0,12 & 0 & & & & & & & & & & & \\
\hline 3 & 0 & 0,1 & 0 & & & & & & & & & & \\
\hline 4 & $-0,03$ & $-0,12$ & 0,32 & 0 & & & & & & & & & \\
\hline 5 & 0,03 & 0,08 & $-0,04$ & 0 & 0 & & & & & & & & \\
\hline 6 & 0 & 0,05 & 0 & 0 & $-0,24$ & 0 & & & & & & & \\
\hline 7 & 0 & 0 & 0,07 & 0,16 & $-0,04$ & 0,27 & 0 & & & & & & \\
\hline 8 & 0 & $-0,09$ & 0 & 0 & 0 & 0,14 & 0 & 0 & & & & & \\
\hline 9 & 0 & 0 & 0 & 0 & 0 & 0,27 & 0,14 & 0,24 & 0 & & & & \\
\hline 10 & 0 & $-0,1$ & 0 & 0 & 0,19 & 0,13 & 0,09 & 0,33 & 0,29 & 0 & & & \\
\hline 11 & 0,08 & 0 & 0 & 0 & 0 & $-0,13$ & 0,07 & 0 & $-0,03$ & 0 & 0 & & \\
\hline 12 & $-0,16$ & $-0,09$ & 0 & 0 & 0 & 0 & 0 & 0,27 & 0 & 0,01 & $-0,36$ & 0 & \\
\hline 13 & 0 & $-0,13$ & 0 & 0 & 0 & 0 & 0 & 0 & 0,21 & 0,01 & $-0,05$ & 0,07 & 0 \\
\hline
\end{tabular}

Tempo 2

\begin{tabular}{|c|c|c|c|c|c|c|c|c|c|c|c|c|c|}
\hline & 1 & 2 & 3 & 4 & 5 & 6 & 7 & 8 & 9 & 10 & 11 & 12 & 13 \\
\hline 1 & 0 & & & & & & & & & & & & \\
\hline 2 & 0,08 & 0 & & & & & & & & & & & \\
\hline 3 & 0 & 0 & 0 & & & & & & & & & & \\
\hline 4 & 0 & 0 & $-0,44$ & 0 & & & & & & & & & \\
\hline 5 & 0 & 0 & 0 & 0 & 0 & & & & & & & & \\
\hline 6 & 0 & 0,03 & 0 & $-0,03$ & 0 & 0 & & & & & & & \\
\hline 7 & 0 & $-0,22$ & 0,05 & 0 & 0 & 0 & 0 & & & & & & \\
\hline 8 & $-0,07$ & $-0,11$ & 0 & 0 & 0 & 0,13 & 0 & 0 & & & & & \\
\hline 9 & 0 & $-0,07$ & 0 & 0 & 0 & 0 & 0 & 0,36 & 0 & & & & \\
\hline 10 & 0 & $-0,1$ & 0 & 0 & 0 & 0 & 0 & 0,2 & 0,32 & 0 & & & \\
\hline 11 & 0 & 0 & 0 & 0 & 0 & $-0,08$ & 0 & $-0,05$ & $-0,1$ & 0 & 0 & & \\
\hline 12 & 0 & 0 & 0,01 & 0 & 0 & 0,14 & 0,02 & 0,35 & 0,06 & 0 & $-0,23$ & 0 & \\
\hline 13 & 0 & $-0,18$ & 0 & 0 & 0 & 0,22 & 0 & 0 & 0,09 & 0 & $-0,07$ & 0 & 0 \\
\hline
\end{tabular}

Nota: 1) Idade, 2) Sexo, 3) Queda de renda, 4) Horas de trabalho, 5) Trabalho na linha de frente de enfrentamento da pandemia, 6) Desesperança percebida, 7) Estigma em relação ao profissional de saúde, 8, 9 e 10) Estresse, Depressão e Ansiedade (DASS-21), 11 e 12) Estratégias de enfrentamento/manejo do estresse Positivas e Negativas e 13) Ideação suicida. 
A Figura 1b apresenta a rede EBIC ( $y=0.50)$ com as associações entre as variáveis medidas no tempo 2. As associações mais expressivas observadas demonstraram haver aumento dos sintomas depressivos mediante o aumento do estresse $(0,36)$ e da ansiedade $(0,32)$; aumento do nivel de estresse quando utilizadas as estratégias de manejo negativo do estresse (0,35); ideações suicidas mais frequentes na presença da desesperança percebida $(0,22)$, e aumento do estresse associado ao aumento da ansiedade $(0,20)$. As associações negativas mais fortes demonstraram que os profissionais da linha de frente trabalharam menos horas semanais $(-0,44)$; a manifestação da percepção negativa de manejo do estresse diminui a expressão da percepção positiva $(-0,23)$; a expressão maior da percepção de estigma em relação ao profissional da saúde aconteceu em pessoas do sexo feminino $(-0,22)$ (ver Tabela 2). As variáveis de maior influência esperada para o segundo período da observação dos profissionais de saúde foram os niveis de estresse, depressão e ansiedade observados pela DASS-21; 1,656, 1,329 e 0,827, respectivamente; a desesperança percebida $(0,773)$ e a percepção negativa de enfrentamento do estresse $(0,667)$ (ver Tabela 6).
A Figura 2 apresenta a rede EBIC ( $y=0.50)$ das associações entre todas as variáveis observadas no tempo 1 e no tempo 2. Para o tempo 1, os resultados indicaram associações mais expressivas, entre o aumento da ansiedade e dos sintomas de depressão e de estresse, variando de 0,21 a 0,31; atuação na linha de frente e mais horas semanais de trabalho $(0,28)$; entre a desesperança, o maior estigma percebido em relação ao profissional de saúde e o aumento da depressão, ambas associações iguais a 0,21, e a diminuição da percepção de enfrentamento positivo e o aumento do estresse $(0,24)$. Entre as associações negativas mais fortes encontram-se a percepção negativa do enfrentamento do estresse e a diminuição do positivo $(-0,32)$, a diminuição da satisfação com a vida e o aumento dos sintomas depressivos $(-0,25)$ e do estigma profissional $(-0,20)$ (ver Tabela 4). As variáveis de maior influência esperada para esse periodo foram o nivel de estresse, a ansiedade e a depressão, 2,026; 1,742 e 1,559 respectivamente; horas de trabalho $(0,443)$; ser um profissional da linha de frente de enfrentamento da COVID-19 $(0,416)$ e a dimensão extroversão da personalidade $(0,336)$ (ver Tabela 5).

a
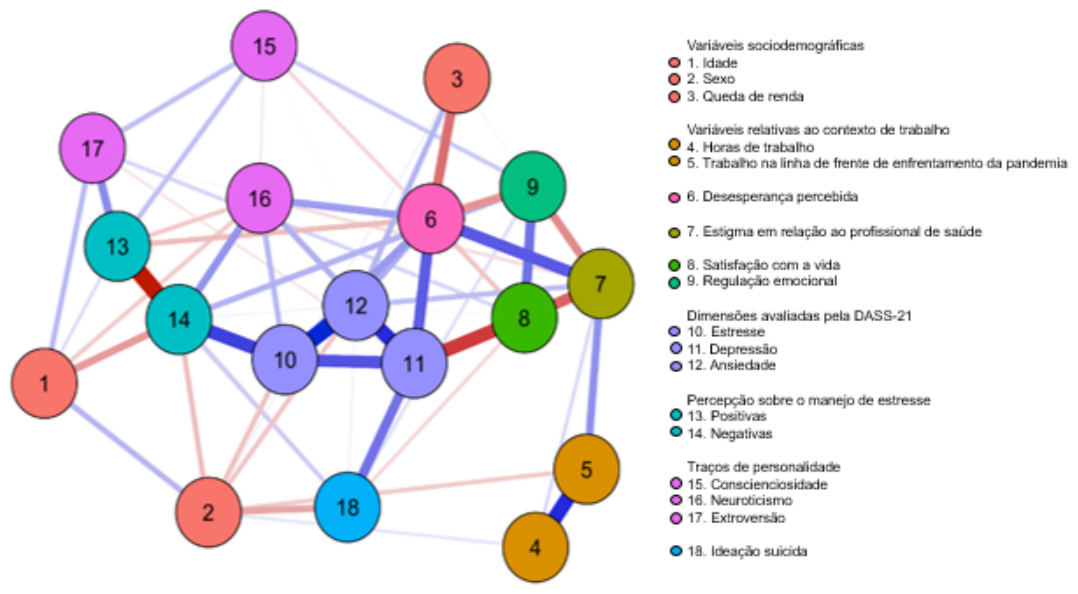


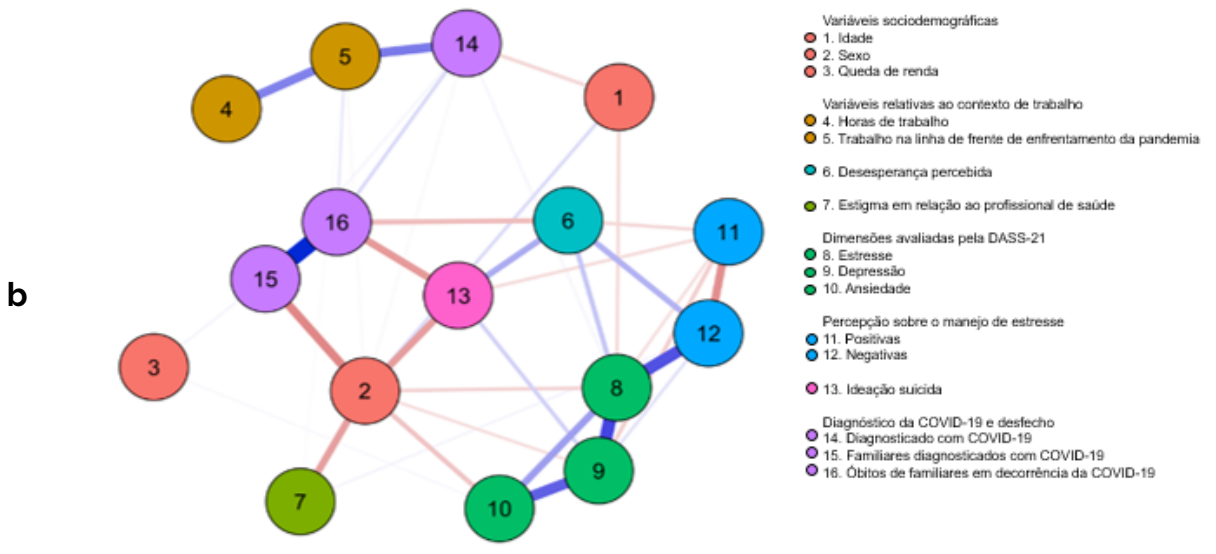

Figura 2 - Rede EBIC y (0.50) das associações entre as variáveis estudadas no Tempo 1 (a) e entre as variáveis estudadas no Tempo 2 (b)

Tabela 4 - Matriz de pesos de todas as variáveis observadas somente no tempo 1 do estudo

\begin{tabular}{|c|c|c|c|c|c|c|c|c|c|c|c|c|c|c|c|c|c|c|}
\hline & 1 & 2 & 3 & 4 & 5 & 6 & 7 & 8 & 9 & 10 & 11 & 12 & 13 & 14 & 15 & 16 & 17 & 18 \\
\hline 1 & 0,00 & & & & & & & & & & & & & & & & & \\
\hline 2 & 0,09 & 0,00 & & & & & & & & & & & & & & & & \\
\hline 3 & 0,00 & 0,02 & 0,00 & & & & & & & & & & & & & & & \\
\hline 4 & 0,00 & 0,03 & 0,00 & 0,00 & & & & & & & & & & & & & & \\
\hline 5 & 0,00 & $-0,07$ & 0,00 & 0,28 & 0,00 & & & & & & & & & & & & & \\
\hline 6 & 0,00 & 0,00 & $-0,18$ & 0,00 & 0,00 & 0,00 & & & & & & & & & & & & \\
\hline 7 & 0,00 & 0,00 & $-0,01$ & 0,05 & 0,14 & 0,21 & 0,00 & & & & & & & & & & & \\
\hline 8 & 0,00 & 0,00 & 0,00 & 0,00 & 0,00 & $-0,07$ & $-0,20$ & 0,00 & & & & & & & & & & \\
\hline 9 & 0,00 & 0,00 & 0,00 & 0,00 & 0,00 & $-0,13$ & $-0,15$ & 0,18 & 0,00 & & & & & & & & & \\
\hline 10 & 0,00 & $-0,08$ & 0,00 & 0,00 & 0,00 & 0,13 & 0,00 & 0,00 & 0,00 & 0,00 & & & & & & & & \\
\hline 11 & 0,00 & 0,00 & 0,00 & 0,00 & 0,00 & 0,21 & 0,07 & $-0,25$ & 0,00 & 0,22 & 0,00 & & & & & & & \\
\hline 12 & 0,00 & $-0,07$ & 0,09 & 0,00 & 0,00 & 0,10 & 0,08 & $-0,01$ & 0,00 & 0,32 & 0,27 & 0,00 & & & & & & \\
\hline 13 & 0,04 & 0,00 & 0,00 & 0,00 & 0,00 & $-0,08$ & 0,00 & 0,00 & 0,00 & 0,00 & 0,00 & 0,00 & 0,00 & & & & & \\
\hline 14 & $-0,13$ & $-0,08$ & 0,00 & 0,00 & 0,00 & 0,00 & 0,00 & 0,00 & 0,10 & 0,24 & 0,00 & 0,01 & $-0,32$ & 0,00 & & & & \\
\hline 15 & 0,00 & 0,00 & 0,00 & 0,00 & 0,00 & $-0,05$ & 0,00 & 0,02 & 0,07 & 0,00 & 0,00 & 0,00 & 0,08 & 0,00 & 0,00 & & & \\
\hline 16 & $-0,06$ & 0,00 & 0,00 & 0,00 & 0,00 & 0,12 & $-0,04$ & 0,00 & 0,00 & 0,09 & 0,10 & 0,00 & $-0,07$ & 0,13 & $-0,03$ & 0,00 & & \\
\hline 17 & 0,09 & 0,00 & 0,00 & 0,00 & 0,00 & 0,00 & 0,00 & 0,05 & 0,00 & 0,00 & $-0,03$ & 0,00 & 0,15 & 0,00 & 0,08 & $-0,01$ & 0,00 & \\
\hline 18 & 0,00 & $-0,11$ & 0,00 & 0,00 & 0,00 & 0,00 & 0,00 & $-0,05$ & 0,04 & 0,00 & 0,18 & 0,02 & $-0,04$ & 0,06 & 0,00 & 0,00 & 0,00 & 0,00 \\
\hline
\end{tabular}

Nota: 1) Idade, 2) Sexo, 3) Queda de renda, 4) Horas de trabalho, 5) Trabalho na linha de frente de enfrentamento da pandemia, 6) Desesperança percebida, 7) Estigma em relação ao profissional de saúde, 8) Satisfação com a vida, 9) Regulação Emocional, 10, 11 e 12) Estresse, Depressão e Ansiedade (DASS-21), 13 e 14) Estratégias de enfrentamento/manejo do estresse Positivas e Negativas e 15) Conscienciosidade, 16) Neuroticismo, 17) Extroversão e 18) Ideação suicida 
Para o tempo 2, as associações negativas mais fortes demonstraram que mais pessoas do sexo feminino contrairam a COVID-19 $(-0,24)$; a percepção negativa de enfrentamento do estresse diminui as percepções positivas $(-0,22)$; e menos ideação suicida entre os que perderam algum familiar devido a COVID-19 (-0,20) (ver Tabela 5). As variáveis de maior influência neste modelo foram o nivel de estresse e a depressão (1,555 e 1,213), seguidas pelo trabalho na linha de frente (0,928), a ansiedade (0,590), a percepção de enfrentamento negativo do estresse $(0,476)$, ter tido familiares diagnosticados $(0,385)$ ou que faleceram em decorrência da COVID-19 $(0,335)$ e ter recebido diagnóstico de COVID-19 (0,312) (ver Tabela 6).

Tabela 5 - Matriz de pesos de todas as variáveis observadas somente no tempo 2 do estudo

\begin{tabular}{|c|c|c|c|c|c|c|c|c|c|c|c|c|c|c|c|c|}
\hline & 1 & 2 & 3 & 4 & 5 & 6 & 7 & 8 & 9 & 10 & 11 & 12 & 13 & 14 & 15 & 16 \\
\hline 1 & 0,00 & & & & & & & & & & & & & & & \\
\hline 2 & 0,07 & 0,00 & & & & & & & & & & & & & & \\
\hline 3 & 0,00 & 0,00 & 0,00 & & & & & & & & & & & & & \\
\hline 4 & 0,00 & 0,00 & 0,00 & 0,00 & & & & & & & & & & & & \\
\hline 5 & 0,00 & $-0,01$ & 0,00 & 0,25 & 0,00 & & & & & & & & & & & \\
\hline 6 & 0,00 & 0,00 & 0,00 & 0,00 & 0,00 & 0,00 & & & & & & & & & & \\
\hline 7 & 0,00 & $-0,19$ & 0,00 & 0,00 & 0,01 & 0,00 & 0,00 & & & & & & & & & \\
\hline 8 & $-0,07$ & $-0,09$ & 0,00 & 0,00 & 0,00 & 0,11 & 0,00 & 0,00 & & & & & & & & \\
\hline 9 & 0,00 & $-0,06$ & 0,00 & 0,00 & 0,00 & 0,00 & 0,00 & 0,37 & 0,00 & & & & & & & \\
\hline 10 & 0,00 & $-0,12$ & 0,01 & 0,00 & 0,00 & 0,00 & 0,00 & 0,19 & 0,31 & 0,00 & & & & & & \\
\hline 11 & 0,00 & 0,00 & 0,00 & 0,00 & 0,00 & $-0,08$ & 0,00 & $-0,06$ & $-0,09$ & 0,00 & 0,00 & & & & & \\
\hline 12 & 0,00 & 0,00 & 0,00 & 0,00 & 0,00 & 0,15 & 0,02 & 0,35 & 0,06 & 0,00 & $-0,22$ & 0,00 & & & & \\
\hline 13 & 0,00 & $-0,19$ & 0,00 & 0,00 & 0,00 & 0,16 & 0,00 & 0,00 & 0,09 & 0,00 & $-0,07$ & 0,00 & 0,00 & & & \\
\hline 14 & $-0,08$ & $-0,01$ & 0,00 & 0,00 & 0,26 & 0,00 & 0,00 & 0,02 & 0,00 & 0,00 & 0,00 & 0,00 & 0,00 & 0,00 & & \\
\hline 15 & 0,00 & $-0,24$ & 0,02 & 0,00 & 0,00 & 0,00 & 0,00 & 0,00 & 0,00 & 0,00 & 0,00 & 0,00 & 0,00 & 0,02 & 0,00 & \\
\hline 16 & 0,00 & 0,00 & 0,00 & 0,00 & 0,04 & $-0,13$ & 0,00 & 0,00 & 0,00 & 0,00 & 0,00 & 0,00 & $-0,20$ & 0,07 & 0,51 & 0,00 \\
\hline
\end{tabular}

Nota: 1) Idade, 2) Sexo, 3) Queda de renda, 4) Horas de trabalho, 5) Trabalho na linha de frente de enfrentamento da pandemia, 6) Desesperança percebida, 7) Estigma em relação ao profissional de saúde, 8, 9 e 10) Estresse, Depressão e Ansiedade (DASS-21), 11 e 12) Estratégias de enfrentamento/manejo do estresse Positivas e Negativas, 13) Ideação suicida, 14) Recebeu diagnóstico de COVID-19, 15) familiares com diagnóstico de COVID-19) e 16) óbitos na familia decorrente de COVID-19. 
Tabela 6 - Influência esperada das variáveis mensuradas nos dois momentos do estudo

Influência Esperada

\begin{tabular}{|c|c|c|c|c|}
\hline Variáveis & Tempo $1^{*}$ & Tempo 2* & Tempo 1** & Tempo $2^{* *}$ \\
\hline Idade & -0.566 & -0.079 & -0.486 & -0.533 \\
\hline Sexo & -1.021 & -1.344 & -1.337 & -2.293 \\
\hline Horas de Trabalho & 0.275 & -0.939 & 0.443 & 0.232 \\
\hline Linha de Frente & 0.017 & -1.140 & 0.416 & 0.928 \\
\hline Queda de Renda & -0.700 & -0.099 & -0.799 & -0.265 \\
\hline Desesperança & 0.302 & 0.773 & 0.121 & 0.168 \\
\hline Estigma & 0.867 & -0.416 & -0.142 & -0.723 \\
\hline Estresse & 1.138 & 1.656 & 2.026 & 1.555 \\
\hline Depressão & 1.573 & 1.329 & 1.559 & 1.213 \\
\hline Ansiedade & 1.254 & 0.827 & 1.742 & 0.590 \\
\hline Percepção do Manejo de Estresse Positivo & -1.512 & -1.260 & -1.263 & -1.557 \\
\hline Percepção do Manejo de Estresse Negativo & -1.184 & 0.667 & -0.526 & 0.476 \\
\hline Ideação Suicida & -0.442 & 0.024 & -0.293 & -0.821 \\
\hline Satisfação com a vida & \# & \# & -1.479 & \# \\
\hline Regulação Emocional & \# & \# & -0.289 & \# \\
\hline Conscienciosidade & \# & \# & -0.122 & \# \\
\hline Neuroticismo & \# & \# & 0.094 & \# \\
\hline Extroversão & \# & \# & 0.336 & $\#$ \\
\hline Diagnóstico de Covid-19 & \# & \# & \# & 0.312 \\
\hline Diagnóstico de Covid-19 na família & \# & \# & \# & 0.385 \\
\hline Falecimento por Covid-19 na familia & \# & \# & \# & 0.335 \\
\hline
\end{tabular}

Nota: * influência esperada nos modelos cujas mesmas variáveis foram testadas ** influência esperada nos modelos considerando todas as variáveis observadas no mesmo periodo, e \# variável não mensura.

\section{Discussão}

A discussão apresentada focalizou as variáveis que se mostraram mais influentes nos modelos testados, priorizando as associações entre as variáveis relativas ao contexto da pandemia COVID-19 e as variáveis de saúde mental. Foi possivel perceber fatores que afetaram os profissionais de saúde, para além daqueles aos quais essa população está submetida cotidianamente. Destacou-se a desesperança percebida em relação à pandemia, pois no modelo testado essa variável aumentou sua influência com o avanço da pandemia. Esse efeito pode ser resultante do descontrole da pandemia de COVID-19 no Brasil, caracterizado pela falta de diretrizes, contradições entre as ações governamentais e pelas preocupações com relação à distribuição de insumos e de reforço de profissionais (Henriques \& Vasconcelos, 2020).

A desesperança percebida em relação à pandemia associou-se a maior percepção de estig- 
ma em relação aos profissionais de saúde, e os sintomas depressivos aumentaram mediante à percepção negativa de manejo do estresse. Em estudo realizado por Hacimusalar et al., (2020), para avaliar o quanto profissionais de saúde eram afetados psicologicamente comparados à população em geral durante a pandemia de COVID-19, evidenciou-se que a desesperança era maior entre esses profissionais. Desta forma, destaca-se a relevância desta medida nos protocolos de avaliação e intervenção da saúde mental para esses profissionais no contexto da pandemia de COVID-19. É importante salientar que, mesmo com o controle da pandemia, deve-se pensar os protocolos a serem empregados em contextos futuros, uma vez que situações desconhecidas e envoltas em grandes perdas trazem medo e desesperança (Taylor, 2019).

Ressalta-se o trabalho na linha de frente no enfrentamento da COVID-19 como outro fator que, tanto no tempo 1 quanto no 2 , indicou maior vulnerabilidade dos profissionais de saúde. A atuação na linha de frente no controle desta pandemia foi amplamente descrita como condição associada ao sofrimento mental entre os profissionais de saúde (Lai et al., 2020; Lu et al., 2020). Esses profissionais estão vulneráveis ao estresse devido às condições às quais estão expostos no contexto laboral (Xiao et al., 2020). Algumas destas condições, relatadas por profissionais de saúde que assistiam pacientes com COVID-19, incluiam o acompanhamento dos próprios colegas de trabalho; a falta de EPI e de equipamentos para suporte aos pacientes graves; a ansiedade ao assumir novas atribuições e o aumento das cargas de trabalho (Avanian, 2020).

No presente estudo, profissionais que atuavam na linha de frente tiveram carga horária média de trabalho maior que os demais profissionais no tempo 1. Contudo, no tempo 2, a carga horária do grupo na linha de frente foi menor que a dos demais profissionais. Pode-se pensar que essa mudança quanto à carga horária dos profissionais, decorre do reconhecimento dos riscos e da redução das jornadas de trabalho do grupo que atuava na linha de frente. Além disso, no tempo
2. parte dos profissionais pode ter retomado suas atividades normais de trabalho, interrompidas ou reduzidas no tempo 1, ou adaptado seus atendimentos para o formato remoto, o que explicaria a ampliação da carga horária declarada pelo grupo que não atuava na linha de frente. Na amostra avaliada, houve a participação expressiva de profissionais de psicologia, que coincide com o crescimento da estrutura para atendimentos remotos durante a pandemia no Brasil.

Outra variável, que pode indicar grupos vulneráveis, é o sexo feminino. Observou-se no presente estudo que as mulheres apresentaram mais as formas de enfrentamento negativo de estresse e uma maior percepção de estigmatização por ser profissional de saúde. Outros estudos também apontaram o sexo feminino como variável associada a maiores adversidades no contexto da pandemia de COVID-19, como maiores niveis de ansiedade e de desesperança (Hacimusalar et al., 2020) e mais sintomas depressivos e insônia (Lai et al., 2020).

No tempo 1, a percepção de ser estigmatizado por ser um profissional de saúde foi uma das variáveis com maior influência no modelo testado e foi associada à desesperança, a maior percepção de manejo negativo do estresse, e a menor regulação emocional. Neste momento, a população brasileira encontrava-se em alerta, devido ao desconhecimento quanto ao novo coronavirus e aos relatos do colapso do sistema de saúde e das altas taxas de mortalidade pela COVID-19 na Itália (Pêgo, 2020). Esses fatos poderiam explicar a percepção dos profissionais de saúde quanto à estigmatização. O estigma é tido como um aspecto central para a compreensão da saúde mental dos profissionais de saúde em contextos pandêmicos. Estudos com profissionais de saúde que atuaram na linha de frente, durante o surto de SARS, mostraram que cerca de $20 \%$, em Taiwan (Bai et al., 2004), e de 49\%, em Cingapura (Koh et al., 2005), sentiram-se estigmatizados por causa de seus empregos. Uma das hipóteses para explicar como a estigmatização pode influenciar a saúde mental consiste no possivel efeito mediado pelo estresse que é bem conhecido como um preditor de saúde mental (Lazarus e Folkman, 
1984). Nesta direção, Park et al. (2018) investigaram as relações de estigma e de estresse com a saúde mental em enfermeiras em um hospital de referência, durante epidemia de MERS-CoV, quando essa era uma doença desconhecida na Coréia do Sul, e mostraram que o estigma estava relacionado à saúde mental não apenas direta, mas, também, indiretamente por meio do estresse.

No tempo 2, tanto o estigma quanto a ansiedade mostraram-se menos influentes no modelo, enquanto o estresse e a desesperança tornaram-se mais influentes. No período em que foi realizada a coleta do tempo 2, houve uma ampliação do conhecimento sobre a COVID-19 e a reabertura de serviços, em geral, em boa parte dos estados brasileiros (Pêgo, 2020). Este fato pode explicar as mudanças da influência em relação à estigmatização e à ansiedade na amostra. A manutenção dos sintomas de estresse em profissionais de saúde. por outro lado, foi relatada em estudos anteriores realizados no contexto da pandemia de COVID-19 (Xiao et al., 2020; Lai et al., 2020), bem como em epidemias anteriores (Bai et al., 2004; Park et al., 2018). As variáveis adversas relacionadas ao trabalho, como a exaustão física e mental, a alta taxa de infecção, o medo da contaminação e da transmissão para as pessoas próximas, estão entre os fatores associados ao estresse e outros agravos mentais entre os profissionais de saúde (Lai, 2020; Lu et al., 2020).

Cabe considerar que, no presente estudo, a vivência do óbito de um familiar por COVID-19 associou-se com menor ideação suicida entre os profissionais de saúde no tempo 2. Essa associação, inicialmente contrária ao esperado, deve ser analisada no contexto do público investigado. Profissionais de saúde podem, diante da perda devido à COVID-19, ampliar o senso de responsabilidade no enfrentamento à pandemia distanciando-se dos sentimentos de ideação suicida. Ademais, as equipes profissionais podem ter desenvolvido melhor gerenciamento do desgaste emocional e outros tipos de suporte aos profissionais de saúde. Tal como sugerem Young et al. (2021) o engajamento entre os gestores e os líderes de ambientes hospitalares podem mitigar os aspectos e as experiências pessoais negativas no ambiente de trabalho.

A estruturação dos recursos capazes de reduzir a contaminação, como a oferta de EPIs e treinamentos, foram as principais estratégias voltadas para suporte aos profissionais de saúde durante a pandemia da COVID-19, mas também foram recomendadas intervenções para prevenção e cuidado da saúde mental (Lancet, 2020). Nesta direção, a presente investigação destacou fatores que foram apontados na literatura como relevantes nas intervenções voltadas para saúde mental, como o controle do estresse, a orientação para redução do estigma social e as estratégias de autocuidado e gerenciamento de emoções (Blake et al., 2020).

\section{Considerações finais}

O presente estudo identificou a relevância de fatores compativeis com os indicados nas recomendações para suporte à saúde mental dos profissionais de saúde durante a pandemia COVID-19, como combate à estigmatização destes profissionais, e o tratamento de sintomas depressivos, ansiedade e estresse. O estigma se mostrou uma variável influente nos momentos iniciais da pandemia, contudo a desesperança e o nivel de estresse mantiveram-se expressivos durante o avanço da pandemia. Destacou-se também a necessidade de contemplar fatores já difundidos na literatura que apontam para grupos de maior vulnerabilidade para prejuizos da saúde mental, como o sexo feminino e a atuação na linha de frente no combate à pandemia.

Trata-se de uma pesquisa exploratória e observacional. Sendo assim, estudos quase experimentais ou com amostras randomizadas precisam ser conduzidos para verificar se haverá mudanças produzidas nas variáveis consideradas mais influentes durante a pandemia de COVID-19. Além disso, ressalta-se que a amostra estudada foi não probabilistica e que os resultados foram discutidos com base no contexto geral da pandemia no Brasil, não sendo tratadas situações territoriais de cada região do País. 


\section{Referências}

Andrade, J. M. (2008). Evidências de Validade do Inventário dos Cinco Grandes Fatores de Personalidade para o Brasil [Tese de doutorado, Universidade de Brasília].

Bai, Y., Lin, C. C., Lin, C. Y., Chen, J. Y., Chue, C. M., \& Chou, P. (2004). Survey of Stress Reactions Among Health Care Workers Involved with the SARS Outbreak. Psychiatric Services, 55(9), 1055-1057. https://doi.org/10.1176/appi. ps.55.9.1055

Blake, H., Bermingham, F., Johnson, G., \& Tabner, A. (2020). Mitigating the Psychological Impact of COVID-19 on Healthcare Workers. International Journal of Environmental Research and Public Health, 17(9), Article 2997. https://doi.org/10.3390/ijerph17092997

Caldarelli, G. (2020). A perspective on complexity and networks science. Journal of Physics: Complexity, 1(2), 021001. https://doi.org/10.1088/2632-072X/abga24

Canabarro, A., Tenório, E., Martins, R., Martins, L., Brito, S., \& Chaves, R. (2020). Data-driven study of the COVID-19 pandemic via age-structured modelling and prediction of the health system failure in Brazil amid diverse intervention strategies. PLOS ONE, 15(7), Article e0236310. https://doi.org/10.1371/journal.pone.0236310

Chang, E. C., \& Chang, O. D. (2016). Development of the Frequency of Suicidal Ideation Inventory. Cognitive Therapy and Research, 40(4), 549-556. https://doi. org/10.1007/s10608-016-9758-0

Cohen, S., Kamarck, T., \& Mermelstein, R. (1983). A Global Measure of Perceived Stress. Journal of Health and Social Behavior, 24(4), 385-396. https://doi. org/10.2307/2136404

Diener, E., \& Emmons, R. A. (1985). The Satisfaction with Life Scale. Journal of Personality Assessment, 49, 71-75.

Foygel, R., \& Drton, M. (2010). Extended Bayesian Information Criteria for Gaussian Graphical Models. Advances Neural Information Processing Systems, 23, 2020-2028.

Fruchterman, T. M. J., \& Reingold, E. M. (1991). Graph drawing by force-directed placement. Software: Practice and Experience, 21(11), 1129-1164. https://doi. org/10.1002/spe. 4380211102

Gouveia, V. V., Milfont, T. L., da Fonseca, P. N., \& Coelho, J. A. P. d. M. (2009). Life satisfaction in Brazil: Testing the psychometric properties of the Satisfaction with Life Scale (SWLS) in five Brazilian samples. Social Indicators Research, 90(2), 267-277. https://doi.org/10.1007/ S11205-008-9257-0

Gouveia, V. V., Moura, H. M. de, Oliveira, I. C. V. de, Ribeiro, M. G. C., Rezende, A. T., \& Brito, T. R. de S. (2018). Emotional Regulation Questionnaire (ERQ): Evidence of Construct Validity and Internal Consistency. Psico-USF, 23(3), 461-471. https://doi.org/10.1590/141382712018230306

Gross, J. J., \& John, O. P. (2003). Individual differences in two emotion regulation processes: Implications for affect, relationships, and well-being. Journal of Personality and Social Psychology, 85(2), 348-362. https:// doi.org/10.1037/0022-3514.85.2.348
Hacimusalar, Y., Kahve, A. C., Yasar, A. B., \& Aydin, M. S. (2020). Anxiety and hopelessness levels in COVID-19 pandemic: A comparative study of healthcare professionals and other community sample in Turkey. Journal of Psychiatric Research, 129, 181-188. https:// doi.org/10.1016/j.jpsychires.2020.07.024

Henriques, C. M. P., \& Vasconcelos, W. (2020). Crises dentro da crise: Respostas, incertezas e desencontros no combate à pandemia da Covid-19 no Brasil. Estudos Avançados, 34(99), 25-44. https://doi.org/10.1590/ s0103-4014.2020.3499.003

John, O. P., Donahue, E. M., \& Kentle, R. L. (2012). Big Five Inventory [Data set]. American Psychological Association. https://doi.org/10.1037/t07550-000

Lai, J., Ma, S., Wang, Y., Cai, Z., Hu, J., Wei, N., Wu, J., Du, H., Chen, T., Li, R., Tan, H., Kang, L., Yao, L., Huang, M., Wang, H., Wang, G., Liu, Z., \& Hu, S. (2020). Factors Associated with Mental Health Outcomes Among Health Care Workers Exposed to Coronavirus Disease 2019 JAMA Network Open, 3(3), Article e203976.

Lovibond, S. H., \& Lovibond, P. F. (1996). Manual for the depression anxiety stress scales. Psychology Foundation of Australia.

Langellier, B. A., Yang, Y., Purtle, J., Nelson, K. L., Stankov, l.; \& Diez-Roux, A. V. (2018). Complex Systems Approaches to Understand Drivers of Mental Health and Inform Mental Health Policy. Administration and Policy in Mental Health and Mental Health Services Research, 46(2), 128-144. https://doi.org/10.1007/s10488-018-0887-5

Lu, W., Wang, H., Lin, Y., \& Li, L. (2020). Psychological status of medical workforce during the COVID-19 pandemic: A cross-sectional study. Psychiatry Research, 288, Article 112936. https://doi.org/10.1016/j. psychres.2020.112936

Ministério da Saúde (2020). Boletim Epidemiológico. COE-nCoV. https://portalarquivos2.saude.gov.br/images/ $\mathrm{pdf} / 2020 /$ fevereiro/13/Boletim-epidemiologico-COEcorona-SVS-13fev20.pdf

Moraes, R.F.D. (2021). A Segunda onda da pandemia (mas não do distanciamento físico: Covid-19 e políticas de distanciamento social dos governos estaduais no Brasil. Nota Técnica n. 31. Instituto de Pesquisa Econômica Aplicada. http://repositorio.ipea.gov.br/ handle/11058/10442

Pêgo, B., Moura, R., Nunes, M., Krüger, C., Moreira, P. G., Ferreira, G., \& Nagamine, L. Y. (2020). Nota Técnica n. 16: Pandemia e fronteiras brasileiras: análise da evolução da Covid-19 e proposições. DIRUR - Diretoria de Estudos e Politicas Regionais, Urbanas e Ambientais. IPEA. http:// repositorio.ipea.gov.br/handle/11058/10039

Taylor, S. (2019). The Psychology of pandemics: preparing for the next global outbreak of infectious disease. Cambridge Scholars Publishing.

Teixeira, C. F. de S., Soares, C. M., Souza, E. A., Lisboa, E. S., Pinto, I. C. de M., Andrade, L. R. de, \& Espiridião, M. A. (2020). A saúde dos profissionais de saúde no enfrentamento da pandemia de Covid-19. Ciência \& Saúde Coletiva, 25(9), 3465-3474. https://doi.org/10.1590/1413$\underline{81232020259.19562020}$ 
Teodoro, M. L. M., de Souza, R. B., Martins, C. C., Sediyama, C. Y. N., Alvares-Teodoro, J., Chang, D., \& Chang, E. C. (2020). Validity of the Frequency of Suicidal Ideation Inventory in Brazilian adults. Death Studies, Published online: 24 Sep 2020. https://doi.org/10.1080/07481187.2020.1824201

The Lancet. (2020). COVID-19: Protecting health-care workers. The Lancet, 395(10228), 922. https://doi. org/10.1016/S0140-6736(20)30644-9

Vignola, R. C. B., \& Tucci, A. M. (2014). Adaptation and validation of the depression, anxiety, and stress scale (DASS) to Brazilian Portuguese. Journal of Affective Disorders, 155, 104-109. https://doi.org/10.1016/j. jad.2013.10.031

Xiao, H., Zhang, Y., Kong, D., Li, S., \& Yang, N. (2020). The Effects of Social Support on Sleep Quality of Medical Staff Treating Patients with Coronavirus Disease 2019 (COVID-19) in January and February 2020 in China. Medical Science Monitor, 26, Article eg23549-1. https:// doi.org/10.12659/MSM.923549

Young, K. P., Kolcz, D. L., O'Sullivan, D. M., Ferrand, J., Fried, J., \& Robinson, K. (2021). Health Care Workers' Mental Health and Quality of Life During COVID-19: Results from a Mid-Pandemic, National Survey. Psychiatric Services, 72(2), 122-128. https://doi.org/10.1176/ appi.ps.202000424

\section{Pricila Cristina Correa Ribeiro}

Doutora em Saúde Coletiva pela Universidade do Estado do Rio de Janeiro (UERJ), no Rio de Janeiro, RJ Brasil; professora da Universidade Federal de Minas Gerais (UFMG), em Belo Horizonte, MG, Brasil.

\section{Marco Antônio Silva Alvarenga}

Doutor em psicologia pela Universidade Federal de Minas Gerais (UFMG), em Belo Horizonte, MG, Brasil; professor da Universidade Federal de São João del-Rei (UFSJ), em São João del-Rei, MG, Brasil.

\section{Tiago Geraldo de Azevedo}

Mestrando e Psicólogo pela Universidade Federal de São João del-Rei (UFSJ), em São João del-Rei, MG, Brasil.

\section{Paulo Felipe Ribeiro Bandeira}

Doutor em Ciências do Movimento Humano pela Universidade Federal do Rio Grande do Sul (UFRGS), em Porto Alegre, RS, Brasil; Universidade Regional do Cariri (URCA), em Crato, Ceará, Brasil.

\section{Elder Gomes Pereira}

Mestrando em Cognição e Comportamento pela Universidade Federal de Minas Gerais (UFMG), em Belo Horizonte, MG, Brasil.

\section{Marcela Mansur-Alves}

Doutora em Neurociências pela Universidade Federal de Minas Gerais (UFMG), em Belo Horizonte, MG, Brasil professora da Universidade Federal de Minas Gerais, em Belo Horizonte, MG, Brasil.

\section{Juliana Alvares-Teodoro}

Doutora em Saúde Pública pela Universidade Federal de Minas Gerais (UFMG), em Belo Horizonte, MG, Brasil; professora da Universidade Federal de Minas Gerais, em Belo Horizonte, MG, Brasil.

\section{Camila Batista Peixoto}

Estudante de Graduação em Psicologia da Universidade Federal de Minas Gerais (UFMG), em Belo Horizonte, MG, Brasil.

\section{Marina Luiza Nunes Diniz}

Mestranda em Cognição e Comportamento pela Universidade Federal de Minas Gerais, (UFMG), em Belo Horizonte, MG, Brasil.

\section{Sabrina Kelly Pessoa de Freitas}

Mestranda em Cognição e Comportamento pela Universidade Federal de Minas Gerais (UFMG), em Belo Horizonte, MG, Brasil.

\section{Maycoln Leôni Martins Teodoro}

Doutor em Psicologia Clinica e do Desenvolvimento pela Albert-Ludwigs-Universität Freiburg, Alemanha; professor da Universidade Federal de Minas Gerais (UFMG), em Belo Horizonte, MG, Brasil.

\section{Endereço para correspondência}

Pricila Cristina Correa Ribeiro

Av. Antônio Carlos, 6627

31270-901

Belo Horizonte, MG, Brasil

Os textos deste artigo foram revisados pela Poá Comunicação e submetidos para validação do(s) autor(es) antes da publicação. 